\title{
NITROPRUSSIDE, ITS METABOLITES AND RED CELL FUNCTION
}

\author{
J. du Cailar, * J.C. Mathieu-Daude, ${ }^{*}$ J. Deschodt, ${ }^{*}$ and \\ Y. LAMARCHE† AND C. CASTEL*
}

THE PRESENT STUDY of the biotransformation of sodium nitroprusside (SNP) to sodium thiocyanate $\left(\mathrm{SCN}^{-}\right)$and cyanide $\left(\mathrm{CN}^{-}\right)$was undertaken to evaluate the toxicity of nitroprusside and its metabolites and, in particular, their influence on transport and tissue uptake of oxygen in man.

\section{MATERIALS AND METHODS}

Patients studied. Forty-two patients (20 males and 22 females) undergoing major orthopaedic surgery under general anaesthesia and controlled hypotension were studied. The age range was 12 to 75 years (mean: 36 years $S D=19$ ) and their mean weight $55 \mathrm{~kg}(\mathrm{SD}=14)$.

Hypotensive technique. Hypotension was induced and maintained with a lyophilized solution of SNP 0.01 per cent, using a constant flow mechanical pump. The mean duration of hypotension was 121 minutes $(S D=17)$ with a minimum of 35 minutes and a maximum of 255 minutes. The mean dosage administered to lower mean systemic pressure and to maintain it between 7.32 and $8.65 \mathrm{kPa}$ ( 55 and $65 \mathrm{~mm} \mathrm{Hg}$ ) was $3.22 \mu \mathrm{g} / \mathrm{kg} / \mathrm{min}(\mathrm{SD}=2.12$ ); total dosage varied from $1.5 \mathrm{mg}$ to $70 \mathrm{mg}$, with a mean of $21.30(\mathrm{SD}=$ 17). No tachyphylaxis was encountered.

Anaesthetic management. Neuroleptanalgesia was conducted with the following drugs: a neuroleptic (chlorprotixene), an analgesic (fentanyl) and a sedative hypnotic (diazepam). Tracheal intubation was performed and ventilation was controlled throughout with a volumecycled respirator (RPR). No accidents or complications were encountered during the study.

\section{MeThods \\ Measurements of the following parameters}

* Département d'Anesthésie-Réanimation ( $\operatorname{Pr} \mathrm{J}$. du Cailar, Hôpital Saint Eloi 34059 Montpellier Cedex) et Département de Biophysique Médicale ( $\mathrm{Pr}$. $\mathrm{Ch}$. Benezech-Pr Llory) de la Faculté de Médecine de Montpellier, France.

tDépartement d'Anesthésie-Réanimation, Centre Hospitalier Universitaire, Sherbrooke, Qué., Canada.

Canad. Anaesth. Soc. J., vol. 25, no. 2, March 1978 were done before, during and after the admin istration of SNP: methaemoglobinaemia, cyanmethaemoglobinaemia, carbonic anhydrase, arterial lactate and pyruvate $(n=17)$; arterial and mixed venous blood $\mathrm{PO}_{2}, \mathrm{PCO}_{2}$ and $\mathrm{pH}$, haemoglobin, and haematocrit and, from these, base excess and arterio-venous oxygen difference $\operatorname{Dav}_{\mathrm{O}_{2}}(n=16)$. Before, during (one, two, three and four hours from onset) and at one and two hours after SNP, measurements were made of plasma thiocyanate $\left(\mathrm{SCN}^{-}\right)(\mathrm{n}=42)$, plasma and corpuscular cyanide $\left(\mathrm{CN}^{-}\right)(\mathrm{n}=25)$ and corpuscular 2,3-diphosphoglycerate (2,3-DPG) $(n=$ 17).

Classic techniques were used for measurement of blood gases, acid-base balance, lactate and pyruvate. Plasma thiocyanate was measured with a $\mathrm{SCN}^{-}$specific electrode using the technique described by J.C. Mathieu-Daudé. 1,2 Plasma and corpuscular cyanide were measured by extraction of cyanide in the form of $\mathrm{HCN}$ and colorimetric determination of $\mathrm{CN}^{-}$, an adaptation of the technique of Boxer and Rikards ${ }^{3}$ which we have described in detail elsewhere. ${ }^{4}$ Total blood $\mathrm{CN}^{-}$ was calculated using plasma and corpuscular values and haematocrit.

Corpuscular carbonic anhydrase action was determined spectrophotometrically by measurement of the maximum rate of change of $\left[\mathrm{H}^{+}\right]$in a red blood cell haemolysate during addition of a known quantity of carbon dioxide:

$$
\begin{aligned}
& \text { carbonic anhydrase } \\
& \downarrow \\
& \mathrm{CO}_{2}+\mathrm{H}_{2} \mathrm{O} \rightleftarrows \mathrm{HCO}_{3}^{-}+\mathrm{H}^{+}
\end{aligned}
$$

The V/Vo ratio, normally 25 , was measured. V $=$ rate of change with erythrocytes. $V o=$ rate of change without erythrocyte.

Blood 2,3-DPG levels were measured spectrophotometrically in a red blood cell haemolysate by noting the rate of disappearance of phospho-enol-pyruvate in the presence of enolase and phosphoglycerate mutase, according to the method of Krimski. Using the haematocrit, one can calculate corpuscular 2,3-DPG levels. 
Methaemoglobin and cyanmethaemoglobin were measured spectrophotometrically.

Statistical analysis of data. The statistical significance at $\mathrm{P}<0.05$ of data (mean and standard error of mean $=S E M$ ) presented was studied by a t-test for unpaired data (blood gases, acid-base balance, Dav $\overline{\mathrm{O}}_{02}, 2,3-\mathrm{DPG}$, total $\mathrm{CN}^{-}$) or by a t-test for paired data (carbonic anhydrase, $\mathrm{SCN}^{-}$, lactate and pyruvate).

\section{RESULTS}

Blood gases, acid-base balance and Dav $\overline{0}_{02}$. Table $I$ and Figure 1 show blood gas and acidbase data. No significant variation occurred during perfusion of SNP. After perfusion, an increase in $\mathrm{PaCO}_{2}$ was noted. Arterio-venous oxygen difference is illustrated in Figure 2 and Table Il. Here again no significant variation occurred during SNP perfusion, but there was an increase afterwards.

Methaemoglobin and cyanmethaemoglobin. In none of the cases studied were either methaemoglobin or cyanmethaemoglobin detected at any point.

Corpuscular 2,3-DPG: Table III. No significant variation.

Carbonic anhydrase action. Table IV. No significant variation.

Plasma thiocyanate. Table $\mathrm{V}$ and Figure 3 comparing thiocyanate levels before, during and after SNP administration show an increase of $\mathrm{SNC}^{-}$from an original level of $13.9 \mathrm{mg} / \mathrm{l}( \pm 1.1)$ to $19.2 \mathrm{mg} / \mathrm{l}( \pm 1.3)$ after one hour of SNP to 23.0 $\mathrm{mg} / \mathrm{l}( \pm 2.6)$ at later samplings. After termination of SNP, SCN- levels decrease without reaching the original level for the first two hours. The difference is still significant (paired t-test).

Plasma corpuscular and rotal cyanide. Table VI displays the plasma $\mathrm{CN}^{-}$variations and Table VIl the corpuscular $\mathrm{CN}^{-}$variations. Total $\mathrm{CN}^{-}$, of which corpuscular $\mathrm{CN}^{-}$comprises 90 per cent is shown in Table VIII and Figure 4. During the third hour of SNP perfusion, blood $\mathrm{CN}^{-}$shows a marked rise, from $0.015 \mathrm{mg} / \mathrm{l} \pm 0.002$ before perfusion to $0.300 \mathrm{mg} / \mathrm{l} \pm 0.16$. After SNP is stopped, $\mathrm{CN}^{-}$drops rapidly but after two hours is still significantly elevated $(0.06 \mathrm{mg} / 1 \pm 0.01)$ above the basal value. Note that $\mathrm{CN}^{-}$levels increase steadily during administration of SNP and that serious elevations were seen, reaching $0.700 \mathrm{mg} / \mathrm{l}$ by the third hour in one case and $0.580 \mathrm{mg} / \mathrm{l}$ in another.

Lactate and pyruvate. Table IX and Figure 5 show significant elevations (paired data) of lactate beginning during SNP use and continuing afterwards, the mean value rising from $163 \mathrm{mg} / \mathrm{l}$ \pm 12 to $184 \mathrm{mg} / \mathrm{l} \pm 16$. Variations in pyruvate and lactate-pyruvate ratio are not significant.

\section{Discussion}

Biotransformation, toxicity and dosage of SNP will be discussed in the light of data obtained from the present study.

\section{Thiocyanate Concentration and Toxicity}

Cyanide ions liberated by SNP are believed to be transformed by thiosulfate sulfide transferase, an hepatic enzyme, to thiocyanate. This hypothesis is supported by the progressive increase in $\mathrm{SCN}$ - during the SNP perfusion and its decrease afterwards. The increase is relatively small (mean $9 \mathrm{mg} / \mathrm{l}$ ) with a mean maximum value of $23 \mathrm{mg} / \mathrm{l}$. The results accord with our earlier studies on two series of patients in which mean $\mathrm{SCN}^{-}$values were $18.5 \mathrm{mg} / \mathrm{l}^{4}$ and $29.7 \mathrm{mg} / \mathrm{l}^{2}$ They are lower than those found by Ahearn ${ }^{5}$ during prolonged perfusion (10 to $18 \mathrm{mg} / \mathrm{l}$ ) and slightly higher than those found by Vesey under conditions identical to ours. ${ }^{6}$ According to Page, ? the well-known toxic effects of $\mathrm{SCN}^{-}$appear at $80 \mathrm{mg} / \mathrm{l}$ and become serious at $150 \mathrm{mg} / 1 .^{8}$ Thus the low levels of $\mathrm{SCN}^{-}$formed during controlled hypotension present no risk of toxicity.

Cyanide concentrations and toxicity. It was believed that because of its transformation to $\mathrm{SCN}^{-}$by thiosulfate sulfide transferase (Lang's Rhodanese), $\mathrm{CN}^{-}$ion presented no risk of toxicity. However with the use of high doses $(>3$ $\mathrm{mg} / \mathrm{kg}$ ) numerous workers ${ }^{9-13}$ reported complications associated with $\mathrm{CN}^{-}$ion possibily following accumulation of $\mathrm{CN}^{-}$in the presence of a thiosulfate sulfide transferase deficiency. Results obtained in this study suggest that in man $\mathrm{CN}^{-}$ion is liberated from SNP, accumulates in erythrocytes and is slowly converted to $\mathrm{SCN}^{-}$; as we have seen, $\mathrm{SCN}^{-}$levels remain low even long after perfusion. On the other hand, blood $\mathrm{CN}$ - levels are far from negligible, reaching a mean maximum value of $0.200 \mathrm{mg} / \mathrm{l}$. The $\mathrm{CN}^{-}$concentration increased progressively during perfusion, reading in one case $0.790 \mathrm{mg} / \mathrm{l}$. These results confirm those of Vesey, et al. ${ }^{6}$ and Michenfelder, et $a l .{ }^{14}$ on dogs: the higher values they obtained are explained by higher doses of SNP. Posner ${ }^{15}$ working with monkeys, found $\mathrm{CN}^{-}$levels of 13 $\mathrm{mg} / \mathrm{l}$ after perfusion of $400 \mathrm{mg}$ of SNP over more than two hours.

Thus SNP administration produced significant blood ( 90 per cent corpuscular) $\mathrm{CN}^{-}$levels, vary- 


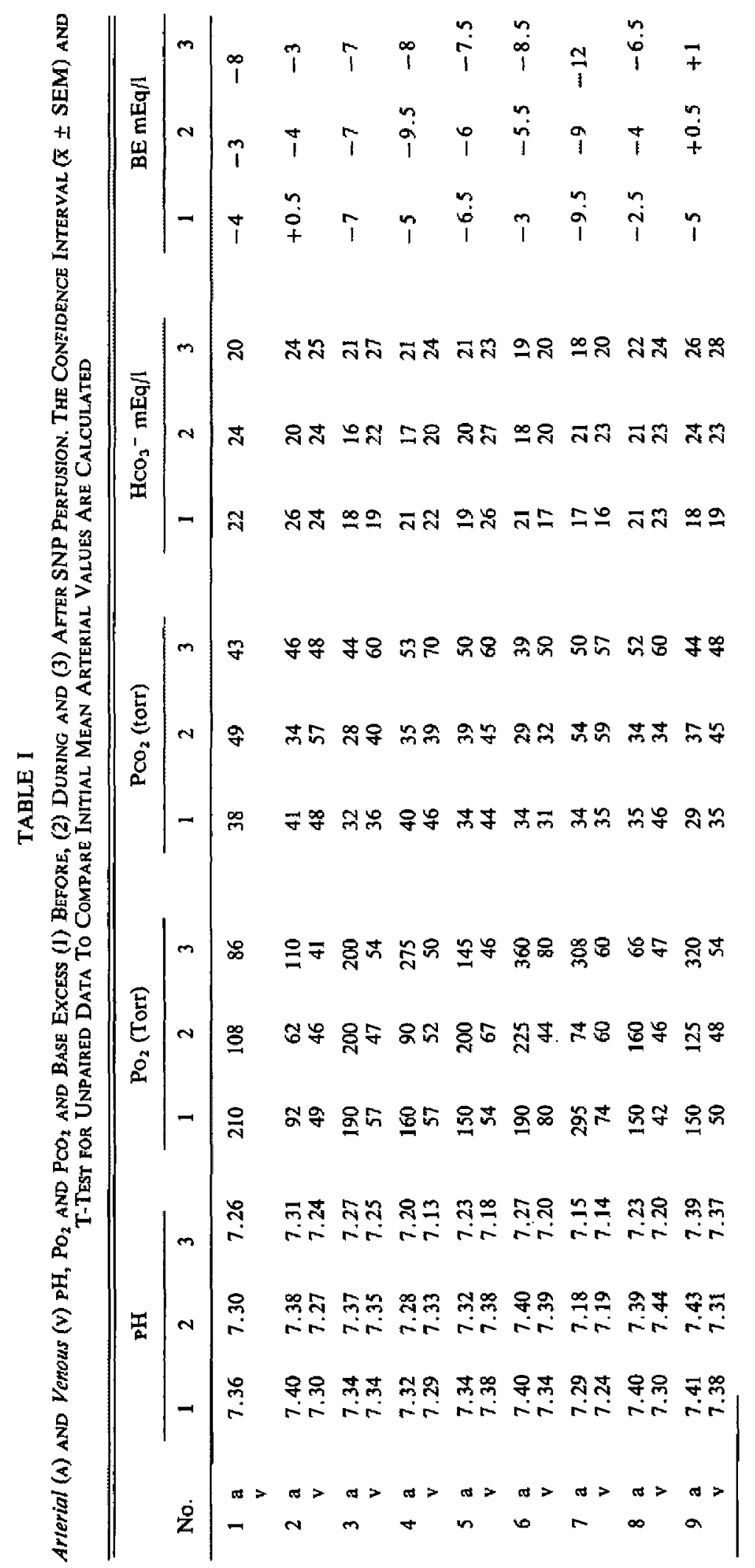




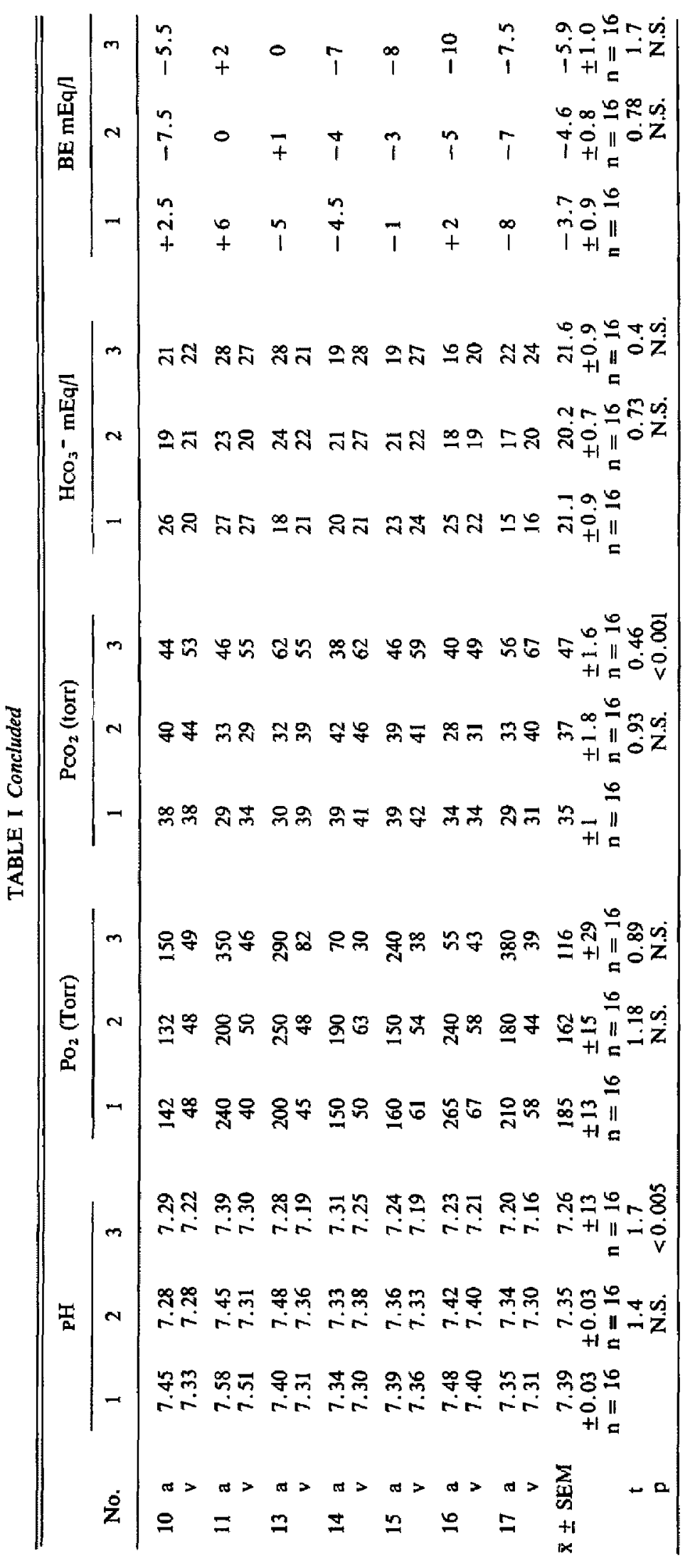




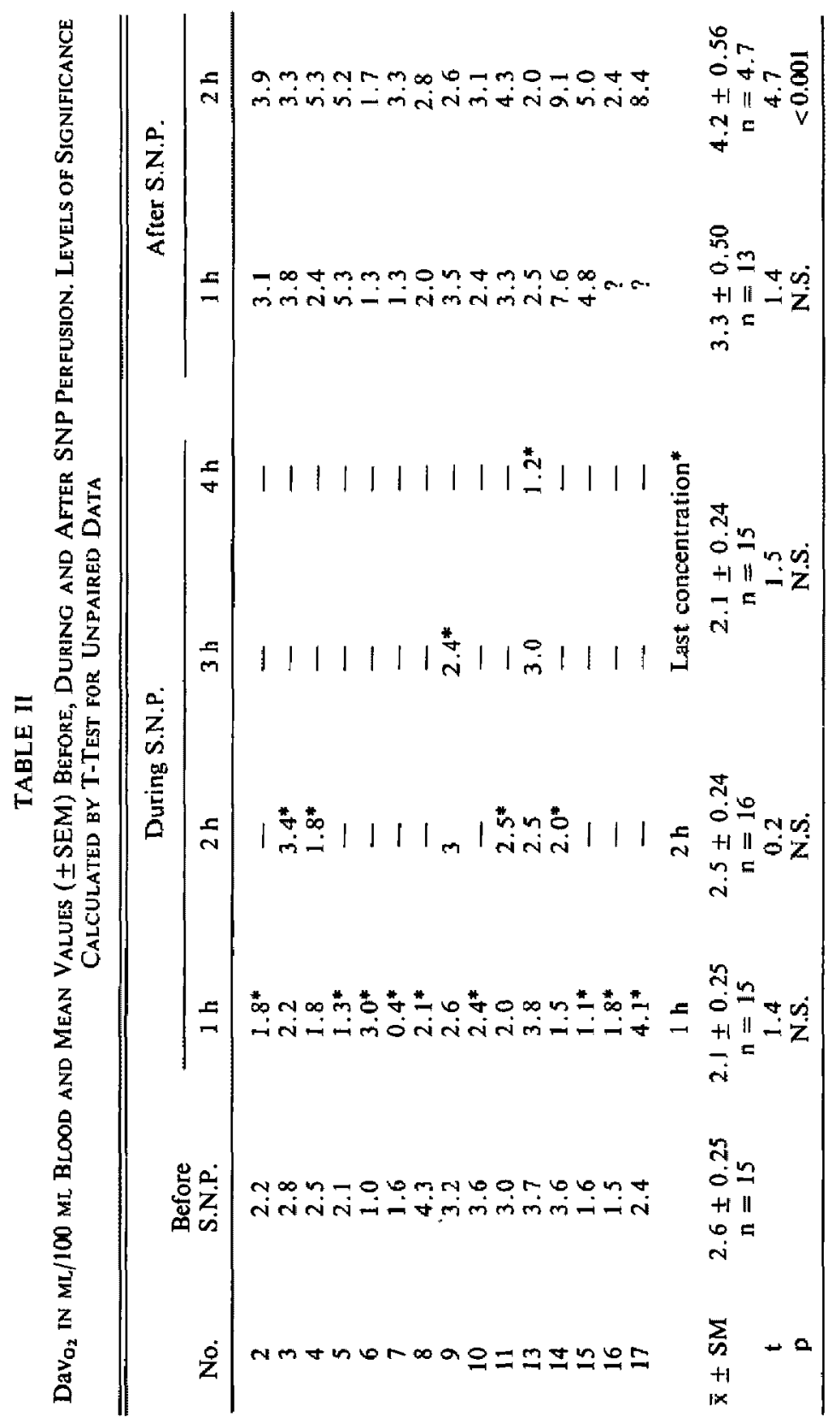


TABLE 111

Corpuscular 2,3-DPG levels in MM/L and Mean Values ( \pm SEM) Before, During and after SNP Perfusion. Levels of Significance Calculated by T-Test for Unpaired Data.

\begin{tabular}{|c|c|c|c|c|c|c|c|}
\hline \multirow[b]{2}{*}{ No. } & \multirow{2}{*}{$\begin{array}{l}\text { Before } \\
\text { S.N.P. }\end{array}$} & \multicolumn{4}{|c|}{ During S.N.P. } & \multicolumn{2}{|c|}{ After S.N.P. } \\
\hline & & $1 \mathrm{~h}$ & $2 \mathrm{~h}$ & $3 \mathrm{~h}$ & $4 \mathrm{~h}$ & $1 \mathrm{~h}$ & $2 \mathrm{~h}$ \\
\hline 2 & 6.42 & 6.42 & $6.35^{*}$ & - & - & & \\
\hline 3 & 5.73 & 5.09 & $5.81^{*}$ & - & - & 5.02 & 5.95 \\
\hline 4 & 5.30 & 4.87 & $5.30^{*}$ & - & - & 4.65 & 6.10 \\
\hline 5 & 3.56 & $3.56^{*}$ & - & - & - & 4.03 & 4.23 \\
\hline 6 & 5.68 & $5.23^{*}$ & - & - & - & 3.87 & 4.31 \\
\hline 7 & 4.63 & $5.05^{*}$ & - & - & - & 5.30 & 5.55 \\
\hline 8 & 6.23 & $6.29^{*}$ & - & - & - & 5.88 & 6.06 \\
\hline 9 & 7.38 & 7.64 & $6.97^{*}$ & - & - & 6.51 & 6.46 \\
\hline 10 & 6.08 & $6.94^{*}$ & - & - & - & 6.88 & 6.88 \\
\hline II & 6.25 & 6.45 & 6.24 & $6.08^{*}$ & - & 5.85 & 5.65 \\
\hline 12 & 6.36 & $6.42 *$ & - & - & - & 5.87 & 5.87 \\
\hline 13 & 6.02 & 6.60 & 6.25 & 6.25 & $7.18^{*}$ & 5.98 & 5.78 \\
\hline 14 & 5.20 & 5.20 & $5.20^{*}$ & & & & \\
\hline 15 & 5.58 & $5.55^{*}$ & - & - & - & 5.56 & 5.32 \\
\hline 16 & 5.32 & $5.60^{*}$ & - & - & - & 5.50 & 5.32 \\
\hline \multirow[t]{2}{*}{17} & 5.54 & $5.41^{*}$ & - & - & - & 4.38 & 4.61 \\
\hline & \multicolumn{7}{|c|}{ Last concentration* } \\
\hline $\bar{x} \pm S E M$ & \multirow[t]{3}{*}{$\begin{array}{c}5.7 \pm 0.22 \\
\mathrm{n}=16\end{array}$} & & \multicolumn{2}{|c|}{$\begin{array}{c}5.8 \pm 0.23 \\
n=16\end{array}$} & & \multicolumn{2}{|c|}{$\begin{array}{c}5.5 \pm 0.20 \\
\mathrm{n}=15\end{array}$} \\
\hline 1 & & & \multicolumn{2}{|c|}{0.4} & & \multicolumn{2}{|c|}{0.5} \\
\hline p & & & \multicolumn{2}{|c|}{ N.S. } & & \multicolumn{2}{|c|}{ N.S. } \\
\hline
\end{tabular}

TABLE IV

Carbonic Anhydrase Activity Before, During and After SNP, V/Vo, Where V = Maximum Rate of Change of $\left(\mathrm{H}^{+}\right)$in the Presence of Erythrocytes, Vo $=$Maximum Rate Without Erthyrocytes. Mean Values \pm SEM. Significance Levels Determined by Paired T-Test

\begin{tabular}{|c|c|c|c|c|c|c|c|}
\hline \multirow[b]{2}{*}{ V/Vo } & \multirow{2}{*}{$\begin{array}{l}\text { Before } \\
\text { S.N.P. }\end{array}$} & \multicolumn{4}{|c|}{ During S.N.P. } & \multicolumn{2}{|c|}{ After S.N.P. } \\
\hline & & $1 \mathrm{~h}$ & $2 \mathrm{~h}$ & $3 \mathrm{~h}$ & $4 \mathrm{~h}$ & $1 \mathrm{~h}$ & $2 \mathrm{~h}$ \\
\hline 1 & 33 & 36 & $37^{*}$ & - & - & 34 & 30 \\
\hline 2 & 34 & $32^{*}$ & - & - & - & 27 & 33 \\
\hline 3 & 29 & 33 & $29^{*}$ & - & - & 34 & 35 \\
\hline 4 & 35 & 37 & $31^{*}$ & - & - & 34 & 29 \\
\hline 5 & 24 & $25^{*}$ & - & - & - & 25 & 26 \\
\hline 6 & 19 & $19^{*}$ & - & - & - & 19 & 19 \\
\hline 7 & 20 & $20^{*}$ & - & - & - & 25 & 23 \\
\hline 8 & 19 & $21^{*}$ & - & - & - & 21 & 20 \\
\hline 9 & 21 & 22 & 22 & $24^{*}$ & - & 22 & 23 \\
\hline 10 & 15 & $15^{*}$ & - & - & - & 14 & 16 \\
\hline 11 & 25 & 26 & $26^{*}$ & - & - & 25 & 26 \\
\hline 12 & 18 & 19 & - & - & - & 20 & 20 \\
\hline 13 & 18 & 20 & 21 & 23 & $23^{*}$ & 26 & 26 \\
\hline 14 & 23 & 20 & $23^{*}$ & - & - & 20 & 19 \\
\hline 15 & 23 & $22^{*}$ & - & - & - & 23 & 25 \\
\hline 16 & 23 & $23^{*}$ & - & - & - & & 21 \\
\hline \multirow[t]{2}{*}{17} & 18 & $19^{*}$ & - & - & - & 20 & 25 \\
\hline & \multicolumn{7}{|c|}{ Last ratio } \\
\hline$\overline{\mathrm{x}} \pm \mathrm{SEM}$ & $\begin{array}{c}23.5 \pm 1.5 \\
\mathrm{n}=17\end{array}$ & $\begin{aligned} 24 & \pm 1.6 \\
\mathrm{n} & =16\end{aligned}$ & & $\begin{array}{r}24 \pm 1 \\
\mathrm{n}=17\end{array}$ & & $\begin{array}{c}24 \pm 1.4 \\
n=17\end{array}$ & $\begin{array}{c}24.5 \pm 1.3 \\
n=17\end{array}$ \\
\hline$t$ & & 1.8 & & 1.4 & & 1.6 & 1.3 \\
\hline p & & N.S. & & N.S. & & N.S. & N.S. \\
\hline
\end{tabular}


TABLE $V$

Plasma Thiocyanate in mg/L and Mean Values (+SEM) Before, During and After SNP, Significance Levels Established by Patred T-Test

\begin{tabular}{|c|c|c|c|c|c|c|c|}
\hline & \multirow{2}{*}{$\begin{array}{l}\text { Before } \\
\text { S.N.P. }\end{array}$} & \multicolumn{4}{|c|}{ During S.N.P. } & \multicolumn{2}{|c|}{ After S.N.P. } \\
\hline & & $1 \mathrm{~h}$ & $2 \mathrm{~h}$ & $3 \mathrm{~h}$ & $4 \mathrm{~h}$ & $1 \mathrm{~h}$ & $2 \mathrm{~h}$ \\
\hline 1 & 10 & & $38 *$ & - & - & 29 & 23 \\
\hline 2 & 13 & $23^{*}$ & - & - & - & 22 & 19 \\
\hline 3 & 20 & 27 & $23^{*}$ & - & - & 21 & 26 \\
\hline 4 & 15 & 13 & $15^{*}$ & - & - & 13 & 12 \\
\hline 5 & 10 & $12^{*}$ & - & - & - & 11 & 11 \\
\hline 6 & 9 & $13^{*}$ & - & - & - & 11 & 10 \\
\hline 7 & 7 & $11^{*}$ & - & - & - & 9 & 8 \\
\hline 8 & 9 & $11^{*}$ & - & - & - & 10 & 9 \\
\hline 9 & 19 & 22 & 22 & $23^{*}$ & - & 23 & 18 \\
\hline 10 & 9 & $12^{*}$ & - & - & - & 13 & 9 \\
\hline 11 & 14 & 18 & $17^{*}$ & - & - & 12 & 17 \\
\hline 12 & 10 & $12^{*}$ & - & - & - & 16 & 11 \\
\hline 13 & 5 & 17 & 16 & 22 & $18^{*}$ & 15 & 9 \\
\hline 14 & 7 & 9 & 9* & - & - & - & - \\
\hline 15 & 15 & $18^{*}$ & - & - & - & 13 & 14 \\
\hline 16 & 15 & $16^{*}$ & - & - & - & $\ldots$ & 14 \\
\hline 17 & 12 & $12^{*}$ & - & - & - & 12 & 12 \\
\hline 18 & 6 & $9 *$ & - & - & - & 12 & 13 \\
\hline 19 & 11 & 13 & 13 & $12^{*}$ & - & 11 & 12 \\
\hline 20 & 16 & $25^{*}$ & - & - & - & 23 & 17 \\
\hline 21 & 23 & $23^{*}$ & - & - & - & 27 & 25 \\
\hline 22 & 41 & 48 & $46^{*}$ & - & - & 43 & 41 \\
\hline 23 & 18 & 21 & $38^{*}$ & - & - & 23 & 20 \\
\hline 24 & 23 & $31^{*}$ & - & - & - & 26 & 23 \\
\hline 25 & 24 & 31 & $24^{*}$ & 31 & - & 29 & 28 \\
\hline 26 & 20 & $25^{*}$ & - & - & - & 21 & 20 \\
\hline 27 & 14 & $26^{*}$ & - & - & - & 15 & - \\
\hline 28 & 21 & $24^{*}$ & - & - & - & 25 & 24 \\
\hline 29 & 12 & $26^{*}$ & - & - & - & 15 & - \\
\hline 30 & 8 & $10^{*}$ & - & - & - & 9 & - \\
\hline 31 & 13 & 27 & $33^{*}$ & - & - & 26 & 15 \\
\hline 32 & 12 & 31 & $19^{*}$ & - & - & 10 & - \\
\hline 33 & 16 & 21 & 25 & $21^{*}$ & - & 17 & 12 \\
\hline 34 & 16 & $25^{*}$ & - & - & - & 16 & - \\
\hline 35 & 24 & $25^{*}$ & - & - & - & 24 & - \\
\hline 36 & 6 & $12^{*}$ & - & - & - & 8 & - \\
\hline 37 & 14 & 16 & $18 *$ & - & - & 16 & - \\
\hline 38 & 9 & 18 & $15^{*}$ & - & - & 11 & - \\
\hline 39 & 10 & $13^{*}$ & - & - & - & 12 & - \\
\hline 40 & 7 & $10^{*}$ & - & - & - & 7 & - \\
\hline 41 & 6 & $7 *$ & - & - & - & 6 & - \\
\hline \multirow[t]{2}{*}{42} & 17 & $23^{*}$ & - & - & - & 20 & - \\
\hline & & & & \multicolumn{2}{|c|}{$\begin{array}{c}\text { Last } \\
\text { concentra- } \\
\text { tion* }\end{array}$} & & \\
\hline $\begin{array}{c}\bar{x} \pm \text { SEM } \\
t \\
p\end{array}$ & $\begin{array}{c}13.9 \pm 1.1 \\
n=42\end{array}$ & $\begin{array}{c}19.2 \pm 1.3 \\
n=41 \\
7.1 \\
<0.001\end{array}$ & $\begin{array}{c}23.2 \pm 2.6 \\
n=16 \\
3.8 \\
<0.005\end{array}$ & $\begin{array}{c}19.8 \\
\mathrm{n} \\
<\end{array}$ & 12.4 & $\begin{array}{c}17.1 \pm 1.2 \\
\eta=40 \\
4.5 \\
<0.001\end{array}$ & $\begin{array}{c}16.8 \pm 1.4 \\
n=28 \\
3.7 \\
<0.001\end{array}$ \\
\hline
\end{tabular}

ing from $0.023 \mathrm{mg} / \mathrm{l}$ to $0.70 \mathrm{mg} / \mathrm{l}$. What are the toxic effects of such levels? The minimum lethal blood $\mathrm{CN}^{-}$concentration is $3.4 \mathrm{mg} / \mathrm{l}^{16}$ Blood $\mathrm{CN}^{-}$levels in one patient reached a maximum of one-quarter of the lethal concentration, a worri- some observation. Yet perhaps the accepted lethal level ought to be questioned: $3.4 \mathrm{mg} / \mathrm{l}$ was the value found post-mortem in one case of death due to cyanide poisoning. ${ }^{16}$ Usually much higher values are seen, averaging $239 \mathrm{mg} / \mathrm{l}$ in the 52 cases of 

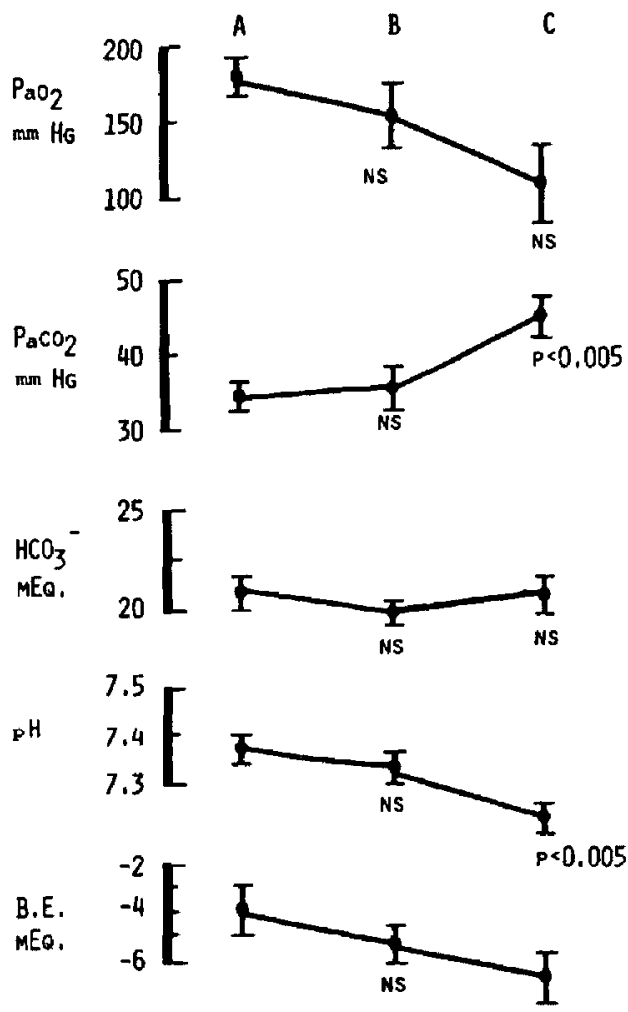

FIGuRE 1 Blood gas and acid-base variations, mean values \pm SEM before (A), during (B) and after (C) SNP perfusion ( $n=16$ ). Significance levels calculated by t-test for unpaired data.

Ansel and Lewis, ${ }^{17}$ Ballantyne et al ${ }^{18}$ have shown that, after death, blood $\mathrm{CN}^{-}$concentration falls rapidly due to diffusion, evaporation, transformation to $\mathrm{SCN}^{-}$, hydrolysis to ammonium formiate and reaction with aldehydes and polysulfides. Curry ${ }^{19}$ reported long ago $\mathrm{CN}^{-}$ concentrations of $0.5 \mathrm{~g} / \mathrm{l}$ to $1.0 \mathrm{~g} / \mathrm{l}$ on the day of death by $\mathrm{CN}^{-}$intoxication. These values are far from the lethal concentration of $3.4 \mathrm{mg} / \mathrm{l}$ suggested by Getler and Baine. ${ }^{16}$ This would explain why neither Vesey ${ }^{6.20 .21}$ or us encountered any clinical signs of intoxication after prolonged SNP perfusion causing blood $\mathrm{CN}^{-}$concentrations above the supposedly lethal levels. Alternatively, as Vesey et $a^{22}$ have suggested, it may be that toxicity is dependent on plasma $\mathrm{CN}^{-}$concentrations, which in our study stayed well below the level $(2.7 \mathrm{mg} / \mathrm{l})$ supposed by Vesey ${ }^{6}$ to be toxic, rather than on corpuscular concentration. There remains, however, the problem of interaction between $\mathrm{CN}^{-}$and haemoglobin.

\section{Cyanide and Haemoglobin}

The high corpuscular $\mathrm{CN}^{-}$concentration can be explained easily if we consider the fact that the principal metabolic pathway of SNP, according to Smith and Kruszyna, ${ }^{23}$ consists of a reaction with free or intracellular haemoglobin by a nonenzymatic oxidation and transfer of a ferrous ion from haemoglobin to SNP, with subsequent formation of methaemoglobin and unstable SNP. From this unstable radical, five $\mathrm{CN}^{-}$ions are liberated and one of these reacts with haemoglobin to form cyanmethaemoglobin.

In the present study, neither methaemoglobin nor cyanmethaemoglobin were detected. This does not weaken the hypothesis of Smith and Kruszyna ${ }^{23}$ in any way, small quantities of these compounds being undetectable spectrophotometrically with the dosage of SNP used. On the other hand, at higher dosages, it is quite probable

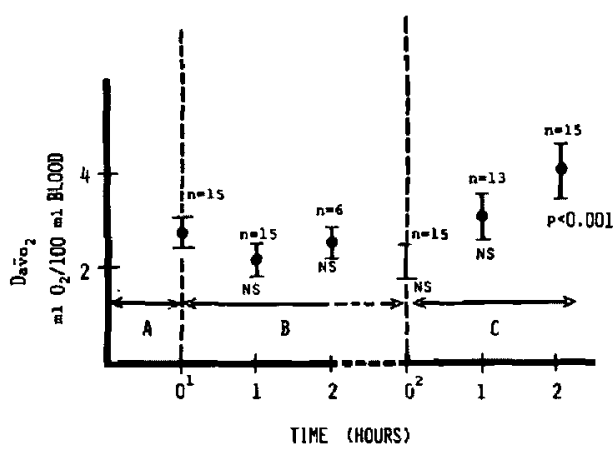

Figure 2 Confidence interval of mean ( \pm SEM) Da $\bar{v}_{0_{2}}$ values in $\mathrm{ml}$ oxygen $/ 100 \mathrm{ml}$ blood before $(\mathrm{A})$ during (B) and after (C) SNP perfusion. Significance calculated by $t$-test for unpaired data to compare with initial values.

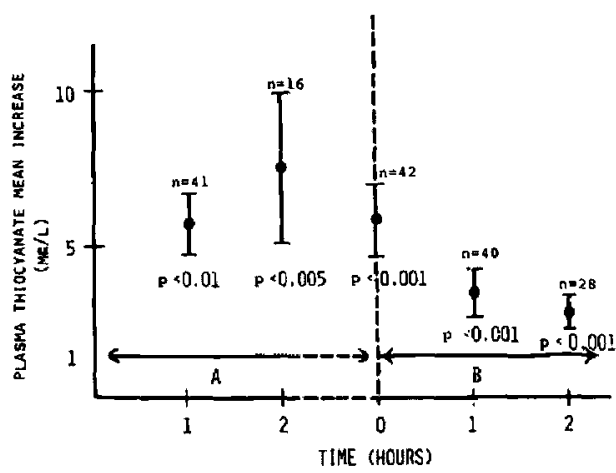

Figure 3 Plasma thiocyanate mean $( \pm S E M)$ increase above initial level during (A) and after (B) SNP. The time of end-perfusion values is displayed at point 0 on the abscissa. 
TABLE VI

Plasma Cyanide in mg/L Before, During and after SNP

\begin{tabular}{|c|c|c|c|c|c|c|c|}
\hline & \multirow{2}{*}{$\begin{array}{l}\text { Before } \\
\text { S.N.P. }\end{array}$} & \multicolumn{4}{|c|}{ During S.N.P. } & \multicolumn{2}{|c|}{ After S.N.P. } \\
\hline & & Ih & $2 \mathrm{~h}$ & $3 \mathrm{~h}$ & $4 h$ & $1 \mathrm{~h}$ & $2 \mathrm{~h}$ \\
\hline 1 & 0,002 & 0.004 & 0.002 & - & - & 0.004 & 0.02 \\
\hline 2 & 0.001 & 0.008 & - & - & - & 0.004 & 0.001 \\
\hline 3 & 0.000 & 0.001 & 0.005 & - & - & 0.004 & 0.001 \\
\hline 4 & 0.003 & 0.006 & 0.006 & - & - & 0.004 & 0.002 \\
\hline 5 & 0.001 & 0.021 & - & - & - & 0.004 & 0.002 \\
\hline 6 & 0.007 & 0.012 & - & - & - & 0.013 & 0.005 \\
\hline 7 & 0.003 & 0.003 & - & - & - & 0.004 & 0.003 \\
\hline 8 & 0.002 & 0.002 & $\ldots$ & - & - & 0.002 & 0.002 \\
\hline 9 & 0.025 & 0.026 & 0.082 & 0.051 & - & 0.032 & 0.026 \\
\hline 10 & 0.008 & 0.068 & - & - & - & 0.006 & 0.006 \\
\hline 12 & 0.010 & 0.013 & - & - & - & 0.012 & 0.010 \\
\hline 13 & 0.008 & 0.029 & 0.060 & 0.047 & 0.053 & 0.020 & 0.007 \\
\hline 14 & 0.009 & 0.037 & 0.039 & - & - & 0.010 & 0.009 \\
\hline 15 & 0.008 & 0.025 & - & $\rightarrow$ & - & 0.005 & 0.005 \\
\hline 16 & 0.008 & 0.008 & - & - & - & - & 0.007 \\
\hline 17 & 0.010 & 0.027 & - & - & - & 0.029 & 0.008 \\
\hline 18 & 0.003 & 0.004 & - & - & - & 0.014 & 0.005 \\
\hline 19 & 0.002 & 0.010 & 0.010 & 0.013 & - & 0.004 & 0.002 \\
\hline 20 & 0.004 & 0.006 & - & - & - & 0.008 & 0.004 \\
\hline 21 & 0.002 & 0.014 & - & - & - & 0.013 & 0.008 \\
\hline 22 & 0.014 & 0.023 & 0.025 & - & - & 0.030 & 0.028 \\
\hline 23 & $?$ & 0.135 & 0.156 & - & - & 0.068 & 0.025 \\
\hline 24 & 0.002 & 0.029 & - & - & - & 0.005 & 0.002 \\
\hline 25 & 0.001 & 0.004 & 0.008 & 0.065 & - & 0.003 & 0.003 \\
\hline
\end{tabular}

TABLE VII

Corpuscular Cyanide in mg/L Before, During and After SNP

\begin{tabular}{|c|c|c|c|c|c|c|c|}
\hline & \multirow{2}{*}{$\begin{array}{l}\text { Before } \\
\text { S.N.P. }\end{array}$} & \multicolumn{4}{|c|}{ During S.N.P. } & \multicolumn{2}{|c|}{ After S.N.P. } \\
\hline & & $1 \mathrm{~h}$ & $2 \mathrm{~h}$ & $3 \mathrm{~h}$ & $4 \mathrm{~h}$ & $1 \mathrm{~h}$ & $2 \mathrm{~h}$ \\
\hline 1 & 0.016 & 0.13 & 0.10 & - & - & 0.100 & 0.120 \\
\hline 2 & 0.010 & 0.500 & - & - & - & 0.250 & 0.070 \\
\hline 3 & 0.013 & 0.470 & 0.420 & - & - & 0.135 & 0.090 \\
\hline 4 & 0.008 & 0.160 & 0.340 & - & - & 0.065 & 0.080 \\
\hline 5 & 0.010 & 0.780 & - & - & - & 0.220 & 0.010 \\
\hline 6 & 0.015 & 0.990 & - & - & - & 0.780 & 0.340 \\
\hline 7 & 0.005 & 0.230 & - & - & - & 0.065 & 0.045 \\
\hline 8 & 0.008 & 0.325 & - & - & - & 0.110 & 0.080 \\
\hline 9 & 0.030 & 1.090 & 1.60 & 1.90 & - & 1.70 & 0.390 \\
\hline 10 & 0.060 & 0.450 & - & - & - & 0.080 & 0.060 \\
\hline 12 & 0.110 & 0.860 & - & - & - & 0.820 & 0.350 \\
\hline 13 & 0.030 & 0.310 & 0.680 & 0.640 & 1.400 & 1.000 & 0.430 \\
\hline 14 & 0.030 & 0.050 & 0.150 & - & - & 0.040 & 0.030 \\
\hline 15 & 0.020 & 0.450 & - & - & - & 0.135 & 0.060 \\
\hline 16 & 0.015 & 0.160 & - & - & - & $?$ & 0.040 \\
\hline 17 & 0.020 & 0.175 & - & - & - & 0.060 & 0.023 \\
\hline 18 & 0.015 & 0.156 & - & - & - & 0.450 & 0.310 \\
\hline 19 & 0.007 & 0.047 & 0.100 & 0.230 & - & 0.038 & 0.029 \\
\hline 20 & 0.060 & 0.150 & - & - & - & 0.290 & 0.060 \\
\hline 21 & 0.014 & 0.095 & - & - & - & 0.130 & 0.068 \\
\hline 22 & 0.075 & 0.990 & 1.600 & - & - & 0.940 & $?$ \\
\hline 23 & 0.085 & 0.180 & 0.310 & - & - & 0.365 & 0.470 \\
\hline 24 & 0.031 & $?$ & - & - & - & $?$ & 0.035 \\
\hline 25 & 0.039 & 0.065 & 0.155 & 0.210 & - & 0.180 & 0.060 \\
\hline
\end{tabular}




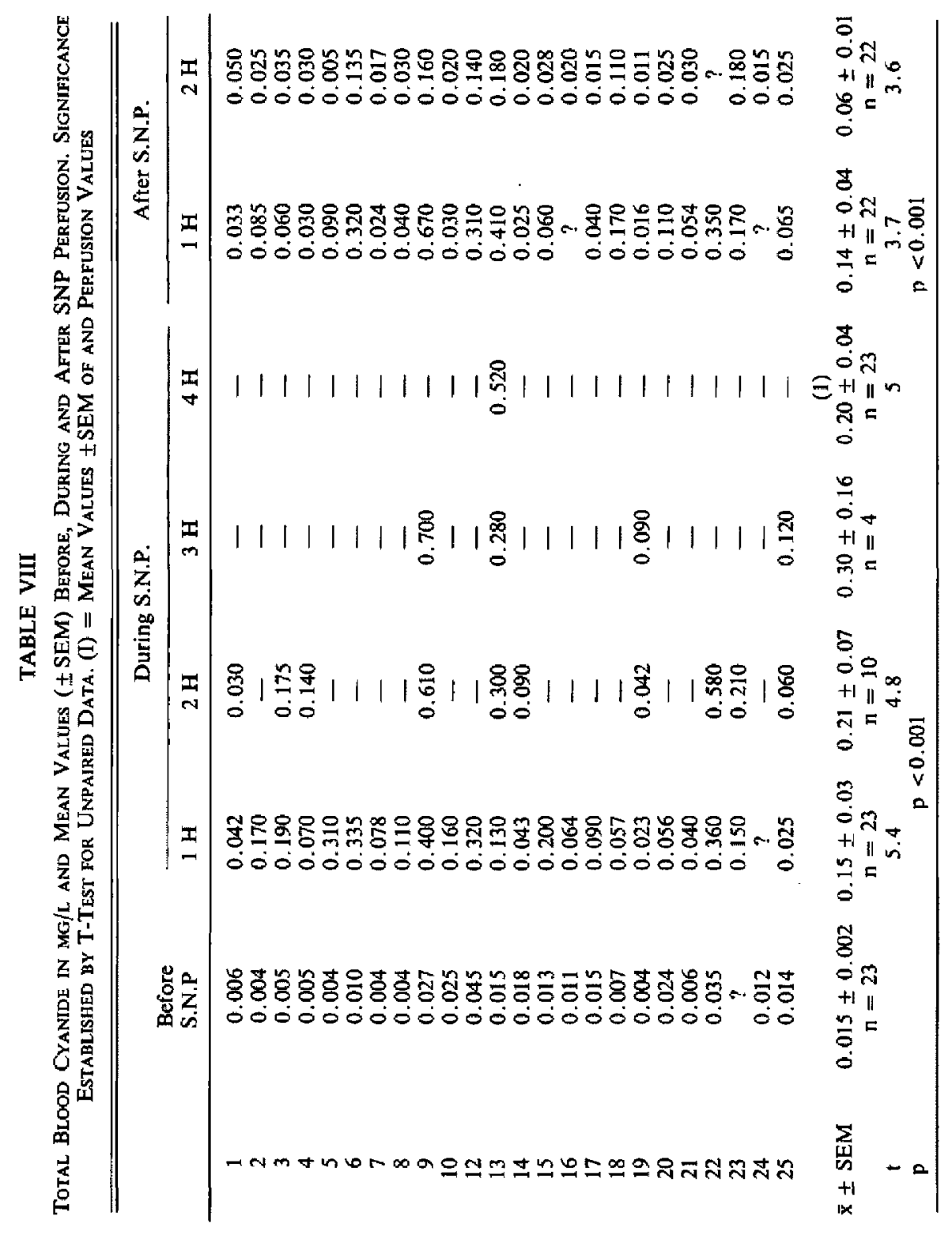




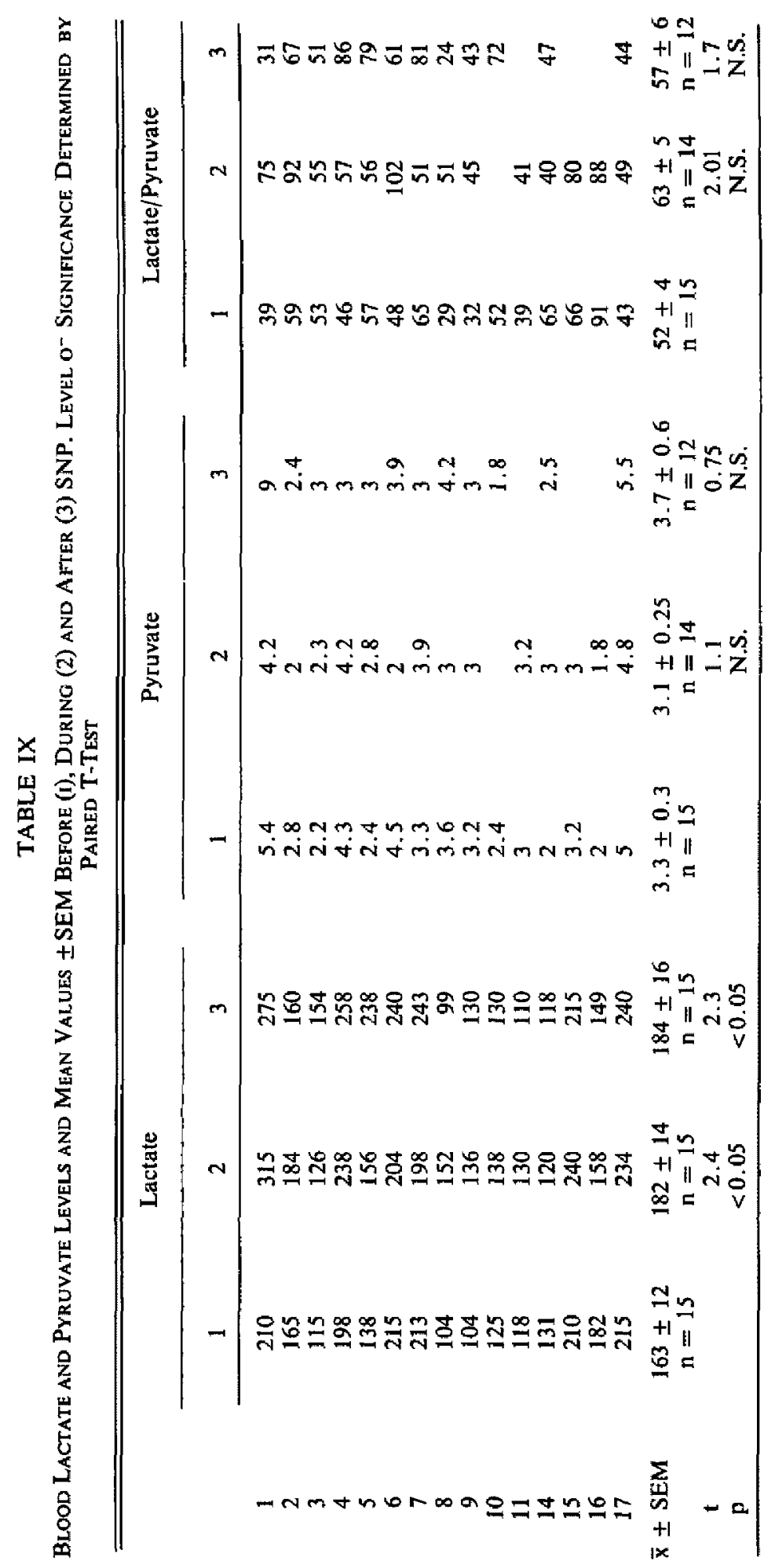




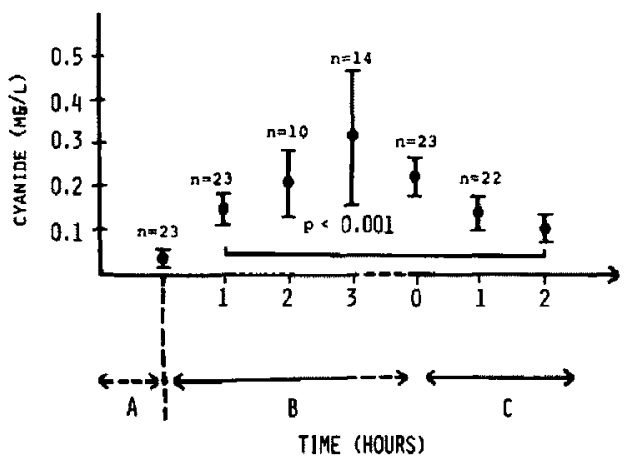

Figure 4 Cyanide in $\mathrm{mg} / \mathrm{l}$ of whole blood before, during and after SNP perfusion. Significance calculated by t-test for unpaired data before (A) during (B) and after (C) SNP. The time of end perfusion values is displayed at point 0 on the abscissa.

that these compounds would be detected. When SNP is administered with methaemoglobin, cyanmethaemoglobin is formed and a larger amount of $\mathrm{CN}^{-}$is carried by the red cell. ${ }^{14}$

Our studies concerning 2,3-DPG and carbonic anhydrase show that the corpuscular $\mathrm{CN}^{-}$has no effect on red cell functions. In vitro studies (Figure 6) have confirmed that interference of $\mathrm{CN}^{-}$with carbonic anhydrase occurs only above dosages used clinically.
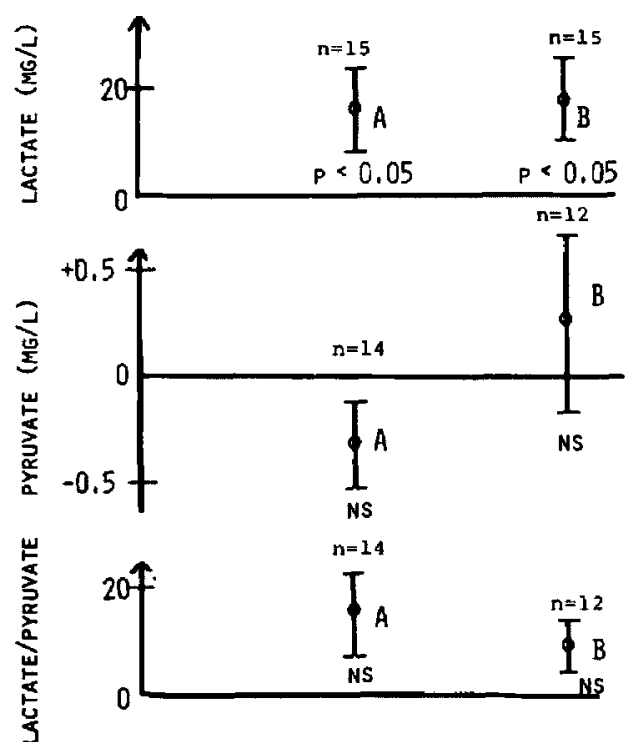

Figure 5 Lactate, pyruvate increase over initial levels and lactate/pyruvate ratio, before, during and after SNP, significance calculated by paired t-test.
Blood gases, Dañ ${ }_{0}$, acid-base balance. Changes in blood gases, $\mathrm{Da} \overline{\mathrm{v}}_{\mathrm{O}_{2}}$, and acid-base balance were reported during clinical use of SNP. A fall of $\mathrm{Pa}_{\mathrm{O}_{2}}$ was observed by Willsmith, et al. ${ }^{24}$ and Francois, et al..$^{25}$ Our past ${ }^{2,4}$ and present studies did not confirm this finding. According to Willsmith, et al. ${ }^{24}$ this decrease in $\mathrm{Pa}_{0,2}$ is caused by an increase of pulmonary shunting by modification of pulmonary vascular resistance due to SNP. It is possible that the strong adrenolytic action of chlorprotixene used as an anaesthetic agent could have prevented any action of SNP on pulmonary vasculature. $\mathrm{PaCO}_{2}$ was increased after SNP. This could be explained by the fact that blood samples were taken during the recovery period at a time when patients already disconnected from the respirator were hypoventilating. $\mathrm{PaCO}_{2}$ was therefore increased and $\mathrm{pH}$ concomitantly decreased.

A decrease of $\operatorname{Dav}_{\mathrm{O}_{2}}$ was noted by many authors during administration of large doses of SNP. ${ }^{8,12,26.27}$ It is believed to be caused by the action of the metabolites of SNP on oxidative metabolism. We did not find this variation during our study. The only significant variation occurred during recovery from anaesthesia when an increase of $\mathrm{Da}_{\mathrm{O}_{2}}$ can be interpreted as a result of returning normal metabolic activity. Likewise, we did not find the metabolic acidosis reported by others. ${ }^{8.11,12}$ On the other hand, we found a slight increase in blood lactate, statistically significant

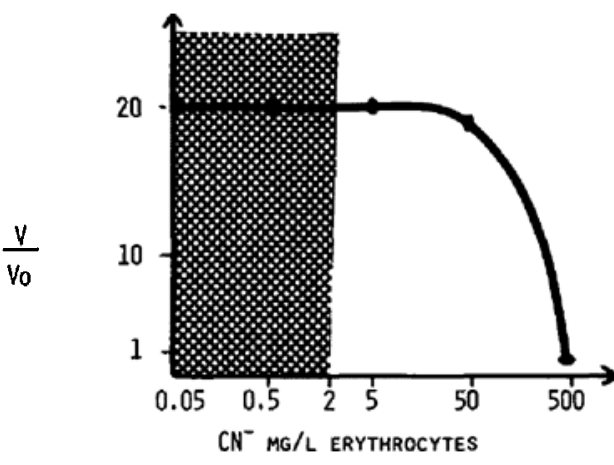

FIGURE 6 In vitro inhibition of corpuscular carbonic anhydrase activity by cyanide $\mathrm{V} / \mathrm{Vo}=$ ratio of the maximum rate of change of $\left[\mathrm{H}^{+}\right]$when a known quantity of $\mathrm{CO}_{2}$ is added to a solution containing erythrocytes (V) and not containing erythrocytes (Vo). The curve obtained shows that under experimental conditions a significant decrease of corpuscular carbonic anhydrase activity occurs when a concentration of at least $50 \mathrm{mg} / \mathrm{l}$ of $\mathrm{CN}^{-}$is reached, more than 25 times the maximum value found during clinical practice (hatched area). 
by the test for unpaired data. With large doses of SNP, severe metabolic acidosis has been reported and is believed to be caused by the appearance of anaerobic metabolism. ${ }^{15.26}$ Although it is slight in our study and not evidently caused by SNP, we consider it an alarming sign.

\section{Maximum SNP Dosage}

In conclusion it appears that at a mean dose of $0.35 \mathrm{mg} / \mathrm{kg}$ administered over two hours, while SNP causes appreciable corpuscular $\mathrm{CN}^{-}$concentrations, it leads to no alteration in red cell function or serious metabolic problems. This is not surprising, since almost all the $\mathrm{CN}^{-}$produced by SNP appears in the blood either as $\mathrm{SCN}^{-}$or as intracorpuscular $\mathrm{CN}^{-}$. Thus, the toxic effects would appear only after corpuscular saturation, elevated plasma levels and tissue fixation of $\mathrm{CN}^{-}$ which, by its action on cytochrome oxidase, interferes with cellular metabolism. Since our study does not allow us to define the maximum tissue fixation of $\mathrm{CN}^{-}$we cannot delimit precisely the maximum allowable dose of SNP, which is dependent on tissue $\mathrm{CN}^{-}$fixation more than on duration of perfusion, itself not a negligible factor. However, we can say that the complications noted in anaesthesia all involved dosages over 3 $\mathrm{mg} / \mathrm{kg}$ and usually over $300 \mathrm{mg} \mathrm{SNP}$. It is widely recognized that such doses are excessive and by their very use betray inadequate activity of the drug and its metabolism. We have never, even for perfusions lasting over three hours, had to use over $70 \mathrm{mg}$, our mean dose being $21 \mathrm{mg}$. Recall that the lethal dose of $\mathrm{CN}^{-}, 3 \mathrm{mg} / \mathrm{kg}$, reflects a SNP dosage of $7 \mathrm{mg} / \mathrm{kg}$. Given the dosages commonly used clinically, we recommend that no more than one-sixth of this dose $(1.16 \mathrm{mg} / \mathrm{kg})$ be used within a limited time period. It appears very difficult to determine this time period. In accordance with usual duration of controlled hypotension, we can set this time limit at two hours. The maximum dosage would be, under these conditions, $0.60 \mathrm{mg} / \mathrm{kg} / \mathrm{hr}$ or $10 \mu \mathrm{g} / \mathrm{kg} / \mathrm{min}$. This value, which we have proposed in an earlier work, ${ }^{2,4}$ is close to that recommended by other authors and in particular by Vesey. ${ }^{6}$

\section{SUMMARY}

The effects on metabolism and red cell function of blood levels of thiocyanate $\left(\mathrm{SCN}^{-}\right)$and cyanide $\left(\mathrm{CN}^{-}\right)$were studied in 42 patients undergoing surgery under controlled hypotension $(\mathrm{CH})$ induced by sodium nitroprusside (SNP).

The mean dosage of SNP administered was $21.38 \mathrm{mg}(\mathrm{SD}=12)$. The durating of perfusion was 121 minutes $(S D=11)$. All operations were performed under neuroleptanalgesia without complications. No tachyphylaxis was encountered.

Under SNP a slight increase of blood $\mathrm{SCN}^{-}$ (from $13.9 \mathrm{mg} / \mathrm{l} \pm 1.1$ to $23 \mathrm{mg} / \mathrm{l} \pm 2.6$ ) was found. Blood levels of $\mathrm{CN}^{-}$are increased mostly in the red cell, the mean value being $0.300 \mathrm{mg} / 1 \pm 0.10$ for whole blood after two hours of perfusion. This value decreased when perfusion was stopped. All blood samples were negative for methaemoglobin and cyanmethaemoglobin. Carbonic anhydrase activity was not modified, $\mathrm{CN}^{-}$toxicity levels for this enzyme being 50 times higher than those found during our study. 2,3-DPG levels did not vary. Blood gases, acid-base balance and $\mathrm{Da} \overline{\mathrm{O}}_{2}$ did not change significantly, although a slight increase in blood lactate was measured. As shown by this study, appreciable amounts of $\mathrm{CN}^{-}$are detected in blood during SNP perfusion while $\mathrm{SCN}^{-}$stays at relatively low levels. Fortunately most of the $\mathrm{CN}^{-}$released from SNP moves into the red cell and does not alter its functions at clinical concentrations. The low plasma concentration of $\mathrm{CN}^{-}$is not sufficient to cause important metabolic disturbances. However, dosages of SNP higher than those administered during this study could increase the blood and tissue $\mathrm{CN}^{-}$to toxic levels. A toxicity study shows that, during a relatively short period of time, SNP dosage should not exceed $1.16 \mathrm{mg} / \mathrm{kg}$ or a maximum of 10 $\mu \mathrm{g} / \mathrm{kg} / \mathrm{min}$ for a period of two hours.

\section{RéSUMÉ}

Chez 42 patients subissant une intervention chirurgicale sous hypotension contrôlée $(\mathrm{HC})$ au nitroprussiate de sodium (SNP) on a étudié le retentissement des taux sanguins de thiocyanate de sodium $\left(\mathrm{SCN}^{-}\right)$et de cyanure $\left(\mathrm{CN}{ }^{\circ}\right)$ sur les grandes fonctions érythrocytaires et métaboliques.

La posologie de SNP a été en valeur moyenne de $21.38 \mathrm{mg}$ ( $\mathrm{SD}=17$ ) ou de $3.22 \mu \mathrm{g} / \mathrm{kg} / \mathrm{min}$ (SD =12). La durée de la perfusion de SNP a été de $121 \mathrm{~min}(S D=17)$. L'anesthésie était du type neuroleptanalgésie. Aucune tachyphylaxie ni incident n'a été constaté.

Sous l'action de SNP on note une faible élévation de $\mathrm{SCN}^{-}$de $13.9 \mathrm{mg} / /( \pm 1.1)$ à $23 \mathrm{mg} / \mathrm{l}( \pm 2.6)$. Le taux de $\mathrm{CN}^{-}$s'élève essentiellement dans l'érythrocyte, la valeur moyenne dans le sang total étant de $0.300 \mathrm{mg} / \mathrm{l} \pm 0.16$, à la troisième heure de perfusion. Ces valeurs décroissent à l'arrêt de la perfusion. La recherche de méthémoglobine et de cyanmethémoglobine a été négative. L'activité de l'anhydrase carbonique 
érythrocytaire n'est pas modifiée, les taux de $\mathrm{CN}^{-}$toxiques pour cette enzyme étant 50 fois supérieurs à ceux notés dans ce travail. Il n'y a pas de variation du taux de 2.3-DPG érythrocytaire. Aucune modification significative n'a été notée sur les gaz du sang, l'équilibre acidobasique, la $\mathrm{Dav}_{\mathrm{O}_{2}}$. Seule a êté détectée une discrète élévation des lactates.

Ces résultats confirment que la perfusion de SNP s'accompagne d'une concentration sanguine en $\mathrm{CN}^{-}$qui est loin d'être négligeable, aiors que celle de $\mathrm{SCN}^{-}$est relativement faible. Heureusement l'essentiel du $\mathrm{CN}^{-}$apporté par SNP est libéré et stocké dans le globule rouge dont il ne modifie pas aux concentrations clinjques notées dans ce travail la fonction. La faible concentration du $\mathrm{CN}^{-}$plasmatique n'est pas suffisante pour entrainer des troubles sérieux du fonctionnement métabolique. Il est cependant prévisible que des concentrations de SNP plus importantes que celles utilisées dans ce travail peuvent entrainer des taux de $\mathrm{CN}^{-}$sanguins et tissulaires toxiques. L'étude de ces concentrations toxiques montre qu'il y a intérêt à ne pas dépasser une posologie de SNP, dans un court laps de temps de $1.16 \mathrm{mg} / \mathrm{kg}$, soit pour une perfusion de deux heures un maximum de 10 $\mu \mathrm{g} / \mathrm{kg} / \mathrm{min}$.

\section{REFERENCES}

1. Mathieu-Daude, J.CL. Etude physico-chimique des électrodes spécifiques. Application à la mesure du calcium ionisé du sérum. Thèse en Médecine, Montpellier (1975).

2. Callar, J.du, Mathieu-Daude, J.Cl., \& DeSCHODr, J. Etude du taux plasmatique et érythrocytaire de l'ion cyanure au cours de la perfusion de nitroprussiate de sodium. Ann. Anesth. Franc. 17: 169 (1976)

3. Boxer, G.E. \& Rickaros, J.C. Chemical determination of vitamine $B_{12}$. The quantitative isolation and colorimetric determination of milliminogram quantities of cyanide. Arch. Biochem. Biophys. 30: 372 (1950).

4. Callar, J.du, Mathieu-Daude, J.Cl., DeSCHODT, J., \& GRIFFE, O. Etude du taux plasmatique de I'ion thiocyanate au cours de la perfu. sion de nitroprussiate de sodium. Ann. Anesth. Franc. 17:519 (1976).

5. AHEARN, D.H. \& GRIM, C.E. Treatment of malignant hypertension with sodium nitroprussiate. Arch. Intern. Med. 133: 187 (1974).

6. Vesey, C.J., Cole, P.V., \& Simpson, P.J. Cyanide and thiocyanate concentrations following sodium nitroprusside infusion in man. Brit. $J$ Anaesth. 48: 65 (1976).

7. Page, I.H., Corcoran, A.C., Dustan, H.P., \& Koppanye, T. Cardio-vascular actions of sodium nitroprusside in animals and hypertensive patients. Circulation $/ 1$ : 188 (1955).
8. Moraca, P.P., BitTe, E.M., Hale, D.E., WasMuth, C.E., \& Poutasse, E.F. Clinical evaluation of sodium nitroprusside as a hypotensive agent. Anesthesiology 23: 193 (1962).

9. Davies, D.W., Kadar, D., Steward, D.J., \& Munro, I.R. A sudden sleath associated with the use of sodium nitroprusside for induction of hypotension during anaesthesia. Canad. Anaesih. Soc. J. $22: 547$ (1975).

10. JACK, R.D. Toxicity of sodium nitroprusside. Brit. J. Anaesth. 46:952 (1974)

11. MacCrae, W.R. \& OWen, M. Severe metabolic acidosis following hypotension induced with sodium nitroprusside. Brit. J. Anaesth. 46: 795 (1974).

12. MacDowall, D.G., Keany, N.P., Turner, J.M., LANE, J.R., \& OKUDA, Y. The loxicity of sodium nitroprusside. Brit. J. Anaesth. 46: 327 (1974).

13. Merrifield, A.J.\& Blundell, M.D. Toxicity of sodium nitroprusside. Brit. J. Anaesth. 46: 324 (1974).

14. Michenfelder, J.D. Cyanide release from sodium nitroprusside on the dog. Anesthesiology 46: $196(1977)$.

15. Posner, M.A., Rookey, F.L., \& Tobey, R.E. Nitroprusside induced cyanide poisoning. Anesthe siology 44: 330 (1976).

16. Getrler, A.O. \& Baine, J.O. The toxicology of cyanide. Amer. J. Med. Sci. 195: 182 (1977).

17. Ansel, M. \& Lewis, Fas. A review of cyanide concentrations found in human organs. Forensic Méd. 17: 148 (1970)

18. Ballantyne, B., Bright, J.E., \& Williams, P. The post-mortem rate of transformation of cyanide. Forensic Sci. 3: 171 (1974).

19. Curry, A.S. Cyanide poisoning. Acta Pharmacol Toxicol. 20:29 (1963).

20. Vesey, C.J., Cole, P.V., LinNel, J.L., \& WILson, J. Some metabolic effects of sodium nitroprusside in man. Brit. Med. J. 2: 140 (1974).

21. Vesey, C.J. \& Cole, P.V. Nitroprusside and cyanide. Brit. J. Anaesth. 47: 1115 (1975).

22. Vesey, C.J., Col. E, P.V., \& Simpson, P.J. Nitropruside and cyanide. Brit. J. Anaesth. 49: 395 (1977).

23. Smith, R.P. \& Kruszrna, H. Nitroprusside produces cyanide poisoning via a reaction with hemoglobins. J. Pharmacol. Exp. Ther. 191: 557 (1974).

24. WILDSMITH, J.A.W., MARShaLl, R.L., JeNkINSON, J.L., MacRaE, W.T., \& SCOTT, D.B. Hemodynamic effects of sodium nitro-prusside during nitrous oxide/halothane. Brit. J. Anaesth. 45: 71 (1973)

25. Francois, G., Fabre, J., Sethian, M., Pontin, R. Gouin, F \& LEVY, G. Le nitroprussiate en neuro-chirurgie. A propos de 34 observations. Ann. Anesth. Franç. 18: 169 (1977).

26. Michenfelder, J.D. \& Theye, R.A. Canine systemic and cerebral effects of hypotension induced by hemorrhage, trimethaphan, halothane or nitroprusside. Anesthesiology 46: 188 (1977).

27. Davies, D.W., Greiss, L., Kadar, D., \& STEWARD, D.J. Sodium nitroprusside in children: observations on metabolism during normal and abnormal responses. Canad. Anaesth. Soc. J. 22: 553 (1975). 